\title{
Almost Convergence Property of Generalized Riesz Spaces
}

\author{
Abdul Hamid Ganie ${ }^{*}$, Dowlath Fathima \\ Department of Basic Science, College of Science and Theoretical Studies, Saudi Electronic Universtiy, Abha-M, Saudi Arabia.
}

How to cite this paper: Abdul Hamid Ganie, Dowlath Fathima. (2020) Almost Convergence Property of Generalized Riesz Spaces. Journal of Applied Mathematics and Computation, 4(4), 249-253.

DOI: $10.26855 /$ jamc.2020.12.016

Received: October 25, 2020

Accepted: November 28, 2020

Published: December 23, 2020

*Corresponding author: Abdul Hamid Ganie, Department of Basic Science, College of Science and Theoretical Studies, Saudi Electronic Universtiy, Abha-M, Saudi Arabia.

Email: a.ganie@seu.edu.sa

\section{Abstract}

Quite recently, the sequence space $r^{q}(p)$ has been study in Altay and Başar and is given by

$$
r^{q}(p)=\left\{x=\left(x_{k}\right) \in \omega: \sum_{k}\left|\frac{1}{Q_{k}} \sum_{j=0}^{k} q_{j} x_{j}\right|^{p_{k}}<\infty\right\} ;
$$

with $0<p_{k} \leq H<\infty, H=\sup _{\mathrm{k}} p_{k}$. Also, the characterization of various matrix classes has been given. Also, the significant classes of almost convergent sequence have been studied in Lorentz. Jalal and Ganie have well structured this sequence space to the spaces of almost convergence and characterize some matrix classes concerning to this approach. We aim in this paper to introduce the new generalized sequence spacevia, $r^{q}(s, p)$ of non-absolute type for $s \geq 0$. Some new type of topological properties will be structured. Furthermore, we also examine for characterizing the matrix classes of the form $\left(r^{q}(s, p): f_{\infty}\right),\left(r^{q}(s, p): f\right)$ and $\left(r^{q}(s, p): f_{0}\right)$, where $f_{\infty}, f$ and $f_{0}$ denote respectively the spaces of almost bounded sequences, almost convergent sequences and almost sequences converging to zero.

\section{Keywords}

Infinite Matrices, Almost Convergence, Matrix Transformations

\section{Introduction}

A sequence space is called as a linear space with real or complex sequences. Throughout the paper $\mathbb{N}, \mathbb{R}$ and $\mathbb{C}$ denotes the set of non-negative integers, the set of real numbers and the set of complex numbers respectively. Let $\omega$ denote the space of all sequences (real or complex); $l_{\infty}$ and $c$ respectively denotes the space of all bounded sequences, the space of convergent sequences. By cs and bs, we represent the spaces of all convergent and bounded series, respectively [1].

Let $X$ and $Y$ be two nonempty subsets of $\omega$. Let $A=\left(a_{n k}\right),(n, k \in \mathbb{N})$ be an infinite matrix of real or complex numbers. We write $(A x)_{n}=A_{n}(x)=\sum_{k} a_{n k} x_{k}$. Then $A x=\left\{A_{n}(x)\right\}$ is called the $A$-transform of $x$, whenever $A_{n}(x)=\sum_{k} a_{n k} x_{k}<\infty$ for all $n$. We write $\lim _{n} A x=\lim _{n} A_{n}(x)$. If $x \in X$ implies $A x \in Y$, we say that $A$ defines a matrix transformations from $X$ into $Y$, denoted by $A: X \rightarrow Y$. By $(X: Y)$, we mean the class of all matrices $A$ 
such that $A: X \rightarrow Y$. As in [2, 3], the matrix domain $X_{A}$ of an infinite matrix $A$ in a sequence space $X$ is defined as

$$
X_{A}=\left\{x=\left(x_{k}\right) \in \omega: A x \in X\right\}
$$

Let $S: l_{\infty} \rightarrow l_{\infty}$ be the shift operator defined by $(S x)_{n}=x_{n+1}$ for all $n \in \mathbb{N}$. A Banach limit $\mathrm{L}$ is defined on $l_{\infty}$ as a non negative linear functional such that $L(s x)=L(x)$ and $L(e)=1,(e=(1,1,1, \ldots))[4,5]$. A sequence space is said to be almost convergent to the generalized limit $\alpha$ if all Banach limits of $x$ are $\alpha$ [6,7]. We denote the set of almost convergent sequences by $f$ i.e.,

$$
f=\left\{x \in l_{\infty}: \lim _{m} t_{m n}(x)=\alpha \text { uniformly in } n\right\}
$$

where, $\quad t_{m n}(x)=\frac{1}{m+1} \sum_{j=0}^{m} x_{j+n}, t_{-1, n}=0$ and $\alpha=f-\lim x$.

Nanda [8] has defined a new set of sequences $f_{\infty}$. We call it as the set of all $f$-bounded sequences, that is,

$$
f_{\infty}=\left\{x \in l_{\infty}: \sup _{m n}\left|t_{m n}(x)<\infty\right|\right\} .
$$

It was further analysed briefly in [9], [10].

Let $\left(q_{k}\right)$ be a sequence of positive numbers and let us write $Q_{n}=\sum_{k} q_{k}$ for $n \in \mathbb{N}$. Then the matrix $R^{q}=\left(r_{n k}^{q}\right)$ of the Riesz mean $\left(R, q_{n}\right)$ is given by

$$
r_{n k}^{q}=\left\{\begin{array}{l}
\frac{q_{k}}{Q_{n}} ; 0 \leq k \leq n, \\
0 ; k>n .
\end{array}\right.
$$

The Reisz mean $\left(R, q_{n}\right)$ is regular if and only if $Q_{n} \rightarrow \infty$ as $\left(R, q_{n}\right) n \rightarrow \infty$ and has been further studied in [2].

We denote by $X^{\beta}$, the $\beta$ - deal of a sequence space $X$ and mean the set of all the sequences $x=\left(x_{k}\right)$ such that $x y=\left(x_{k} y_{k}\right) \in C S$ for all $y=\left(y_{k}\right) \in X$ as can be found in [11], [12] and many others.

\section{Main result}

In this section, we define the new generalized Reisz space $r^{q}(s, p)$ and study its various properties.

Following the authors cited in [7], [13], [14], [15], we the define the generalized Reisz space $r^{q}(s, p)$ for $s \geq 0$ as follows:

where $H=\sup _{\mathrm{k}} p_{k}$.

$$
r^{q}(s, p)=\left\{x=\left(x_{k}\right) \in \omega: \sum_{k}\left|\frac{1}{Q_{k}^{s+1}} \sum_{j=0}^{k} q_{j} x_{j}\right|^{p_{k}}<\infty\right\} ;\left(0<p_{k} \leq H<\infty\right) .
$$

Define the sequence $y=\left(y_{k}\right)$ which will be used, by the $R^{q}$-transform of a sequence $x=\left(x_{k}\right)$, that is,

$$
y_{k}=\frac{1}{Q_{k}^{s+1}} \sum_{j=0}^{k} q_{j} x_{j} ; \text { for } k \in \mathbb{N} \text {. }
$$

Note that for $s=0$, we get the results obtained and studied by the author in [16].

Lemma 2.1 [12]: $f \subset f_{\infty}$.

For brevity in notation, we write 


$$
t_{m n}(A x)=\frac{1}{m+1} \sum_{j=0}^{m} A_{n+i}(x)=\sum_{k} a(n, k, m) x_{k}
$$

where, $a(n, k, m)=\frac{1}{m+1} \sum_{j=0}^{m} a_{n+j, k} ;(n, k, m \in \mathbb{N})$.

$$
\text { Also, } \tilde{a}(n, k, m)=\Delta\left[\frac{a(n, k, m)}{q_{k}}\right] Q_{k}^{s+1}=\left[\frac{a(n, k, m)}{q_{k}}-\frac{a(n, k+1, m)}{q_{k+1}}\right] Q_{k}^{s+1} \text {. }
$$

Theorem 2.2 (i): Let $1<p_{k} \leq H<\infty$ for every $k \in \mathbb{N}$. Then $A \in\left(r^{q}(s, p): f_{\infty}\right)$ if and only if there exists an integer $C>1$ such that

$$
\begin{gathered}
\sup _{n, m \in \mathbb{N}} \sum_{k}\left|\tilde{a}(n, k, m) C^{-1}\right|^{p_{k}^{\prime}}<\infty \\
\left\{\left(\frac{a_{n k}}{q_{k}} Q_{k}^{s+1} C^{-1}\right)^{p_{k}^{\prime}}\right\} \in l_{\infty}, \text { for all } n \in \mathbb{N} .
\end{gathered}
$$

(ii) Let $0 \leq p_{k} \leq 1$ for each $k \in \mathbb{N}$. Then $A \in\left(r^{q}(s, p): f_{\infty}\right)$ if and only if

$$
\sup _{n, k, m \in \mathbb{N}}|\tilde{a}(n, k, m)|^{p_{k}}<\infty \text {. }
$$

Proof: Sufficiency: Suppose the conditions (3) \& (4) holds and $x \in r^{q}(s, p)$. Then $\left\{a_{n, k}\right\}_{k \in \mathbb{N}} \in\left[r^{q}(s, p)\right]^{\beta}$ for every $n \in \mathbb{N}$, the $A$-transform of $x$ exists. Using the relation (2) and inequality which holds for any $C>0$ and any two complex numbers $a, b$ that $|a b| \leq C\left\{\left|a C^{-1}\right|^{p^{\prime}}+\mid b^{p}\right\}$ where $p>1$ and $p^{-1}+\left(p^{\prime}\right)^{-1}=1$, we have

$$
\begin{aligned}
\left|t_{m n}(A x)\right| & =\left|\sum_{k} a(n, k, m) x_{k}\right|=\left|\sum_{k} \tilde{a}(n, k, m) y_{k}\right| \leq \sum_{k}\left|\tilde{a}(n, k, m) y_{k}\right| \\
& \leq \sum_{k} C\left\{\left|\tilde{a}(n, k, m) C^{-1}\right|^{p_{k}{ }^{\prime}}+\left|y_{k}\right|^{p_{k}}\right\}
\end{aligned}
$$

Taking sup on both sides, we get $A x \in f_{\infty}$ for every $x \in r^{q}(u, p)$.

Necessity: Suppose that $A \in\left(r^{q}(s, p): f_{\infty}\right)$ and $1<p_{k} \leq H<\infty$ for every $k \in \mathbb{N}$. Then $A x$ exists for every $x \in r^{q}(\mathrm{~s}, p)$ and this implies that $\left\{a_{n, k}\right\}_{k \in \mathbb{N}} \in\left[r^{q}(s, p)\right]^{\beta}$ for every $n \in \mathbb{N}$, the necessity of (4) is immediate. Now, $\sum_{k} a(n, k, m) x_{k}$ exists for each $m, n$ and $x \in r^{q}(s, p)$,the sequences $\{a(n, k, m)\}_{k \in \mathbb{N}}$ define the continuous linear functionals $\psi_{m n}(x)$ on $r^{q}(s, p)$ by $\psi_{m n}(x)=\sum_{k} a(n, k, m) x_{k} ; \quad(n, k, m \in \mathbb{N})$.

Since $r^{q}(s, p)$ is complete space and $\sup _{n, m}\left|\sum_{k} a(n, k, m) x_{k}\right|<\infty$ on $r^{q}(s, p)$, so by uniform bounded principle, there exists $M>0$ such that

$$
\sup _{m, n}\left|\psi_{m n}(x)\right|=\sup _{m, n}\left|a(n, k, m) x_{k}\right|=\sup _{m, n}\left|\tilde{a}(n, k, m) y_{k}\right|<\infty .
$$

This implies that $\sup _{n, m \in \mathbb{N}} \sum_{k}\left|\tilde{a}(n, k, m) C^{-1}\right|^{p_{k}^{p}}<\infty$, which shows the necessity of the condition (3) and the proof of (i) is complete.

Similarly (ii) follows, and the proof is complete.

Theorem 2.3: Let $1<p_{k} \leq H<\infty$ for every $k \in \mathbb{N}$. Then $A \in\left(r^{q}(s, p): f\right)$ if and only if (3), (4) and (5) hold and there is a sequence $\left(\beta_{k}\right)$ of scalars such that

$$
\lim _{m \rightarrow \infty} \tilde{a}(n, k, m)=\beta_{k}
$$

uniformly in $n$, for every $k \in \mathbb{N}$. 
Proof: Sufficiency: Suppose that the conditions (3), (4), (5) and (6) hold and $x \in r^{q}(s, p)$. Then $A x$ exists and we have by (6) that $\left|a(n, k, m) C^{-1}\right|^{p_{k}} \rightarrow\left|\beta_{k} C^{-1}\right|^{p_{k}}$ as $m \rightarrow \infty$, uniformly in $n$ for each $k \in \mathbb{N}$, which leads us with (3) to the inequality

$$
\begin{aligned}
\sum_{j=0}^{k}\left|\beta_{j} C^{-1}\right|^{p_{j}^{\prime}} & =\lim _{m \rightarrow \infty} \sum_{j=0}^{k}\left|\tilde{a}(n, k, m) C^{-1}\right|^{p_{j}^{\prime}} \quad \text { (uniformly in } n \text { ) } \\
& \leq \sup _{n, m \in \mathbb{N}} \sum_{j=0}^{k}\left|\tilde{a}(n, k, m) C^{-1}\right|^{p_{j}^{\prime}}<\infty
\end{aligned}
$$

holding for every $k \in \mathbb{N}$. Since $x \in r^{q}(s, p)$ by hypothesis and $r^{q}(s, p)$ is isomorphic to $l(p)$, we see that $y \in l(p)$. Therefore, it follows from (7) that the series $\sum_{k} \beta_{k} y_{k}$ and $\sum_{k} \tilde{a}(n, k, m) y_{k}$ converges for each $m, n$ and $y \in l(p)$. Now for given $\varepsilon>0$, choose a fixed $k_{0} \in \mathbb{N}$ such that $\left(\sum_{k=k_{0}}^{\infty}\left|y_{k}\right|^{p_{k}}\right)^{\frac{1}{p_{k}}}<\varepsilon$. Therefore, there exists some $m_{0} \in \mathbb{N}$ such that $\left|\sum_{k=0}^{k_{0}}\left[\tilde{a}(n, k, m)-\beta_{k}\right]\right|<\varepsilon$

for every $m \geq m_{0}$, uniformly in $n$. Since (6) holds, it follows that $\left|\sum_{k=k_{0}}^{\infty}\left[\tilde{a}(n, k, m)-\beta_{k}\right]\right|$ is arbitrary small. Hence, we conclude that

$$
\lim _{m} \sum_{k} a(n, k, m) x_{k}=\lim _{m} \sum_{k} \tilde{a}(n, k, m) y_{k}=\sum_{k} \beta_{k} y_{k}
$$

uniformly in $n$. This shows that $A x \in f$, this proves sufficiency.

Necessity: Suppose that $A \in\left(r^{q}(s, p): f\right)$. Then, since $f \subset f_{\infty}$ [by Lemma (2.2)], the necessities of (3) and (4) are immediately obtained from Theorem 2.2. To prove the necessity of (6), consider the sequence

$$
b_{n}^{(k)}(q)=\left\{\begin{array}{cl}
(-1)^{n-k} \frac{Q_{k}^{s+1}}{q_{n}} & , \quad k \leq n \leq k+1 \\
0 & , 0 \leq n<k \text { or } n>k+1
\end{array}\right.
$$

Since $A x$ exists and is in $f$ for each $x \in r^{q}(s, p)$, one can easily see that $A b^{(k)}(q)=\left\{\Delta\left(\frac{a_{n k}}{q_{k}}\right) Q_{k}^{s+1}\right\}_{n \in \mathbb{N}} \in f$ for all $k \in \mathbb{N}$, which proves the necessity of (6). This concludes the proof.

Note that if we let $\beta_{k} \rightarrow 0$ for each $k$, we have following corolory.

Corolory 2.4: Let $1<p_{k} \leq H<\infty$ for every $k \in \mathbb{N}$. Then $A \in\left(r^{q}(s, p): f_{0}\right)$ if and only if (3), (4), (5) and (6) hold.

Deduction 2.5: Choosing s=0, the results so obtained get reduced to what has been obtained in [14].

\section{Conclusion}

The main idea of this paper is to bring out new way of writing the generalized Riesz difference sequence spaces. We have proved various results in this connection which are more general and extensive than the already classical results.

\section{Acknowledgements}

We are very thankful to the reviewers for their comments that improved the presentation of the paper.

\section{References}

[1] Ganie, A. H. and Sheikh, N. A. (2013). On some new sequence space of non-absolute type and matrix transformations, J. Egypt. Math. Soc., 21, 34-40. 
[2] Neyaz, A. S. and Abdul, H. G. (2012). A new paranormed sequence space and some matrix transformations, Acta Math. Acad. Paedagog. Nyiregyhaziensis, 28, 47-58.

[3] Banach, S. (1932). Theỏries des operations linẻaries, Warszawa.

[4] Sheikh, N. A., Jalal, T., and Ganie, A. H. (2013). New type of sequence spaces of non-absolute type and some matrix transformations, Acta Math. Acad. Paedagog. Nyiregyhaziensis, 29, 51-66.

[5] Ganie, A. H. and Sheikh, N. A. (2015). Infinite matrices and almost convergence, Filomat, 29(6), 1183-1188.

[6] Ganie, A. H. and Antesar, A. (2020). Certain sequence spaces using $\Delta$-operator, Adv. Stud. Contemp. Math. (Kyungshang), 30(1), 17-27.

[7] Lorentz, G. G. (1948). A contribution to the theory of divergent series, Acta Math., (80), 167-190.

[8] Abdul, H. G. (2020). Lacunary sequences with almost and statistical convergence, Annals Commu. Maths., 3(1), 46-53.

[9] Nanda, S. (1979). Matrix transformations and almost boundedness, Glasnik Mat., 14(34), 99-107.

[10] Abdul, H. G. (2020). Riesz spaces using modulus function, Int. jour. Math. Models \& Methods in Appl. Sci., 14, 20-23.

[11] Ganie, A. H., Mobin, A., Sheikh, N. A., and Jalal, T. (2016). New type of Riesz sequence space of non-absolute type, J. Appl. Comput. Math., 5(1), 1-4.

[12] Lascarides, C. G., Maddox, I. J. (1970). Matrix transformations between some classes of sequences, Proc. Camb. Phil. Soc., (68), 99-104.

[13] Sheikh, N. A. and Ganie, A. H. (2013). On the space of $\lambda$-convergent sequence and almost convergence, Thai J. Math., 2(11), 393-398.

[14] Altay, B. and Başar, F. (2002). On the paranormed reisz sequence space of non-absolute type, Southeast Asian Bulletin of Math., (26), 701-715.

[15] Ganie, A. H., Lone, S. A., and Akhter, A. (2020). Generalised Ceşaro difference sequence space of non-absolute type, EKSAKTA, 1(2), 147-153.

[16] Jalal, T. and Ganie, A. H. (2009). Almost convergence and some matrix transformation, International Jour. Math. (Shekhar New Series), 1(1), 133-138. 